\title{
Burn Injuries in Elderly-A Retrospective Analysis of a Ten Year Period
}

\author{
Frank Siemers, Eirini Liodaki, Karl L. Mauss, Felix H. Stang, Tobias von Wild, Peter Mailaender
}

Plastic Surgery, University Clinic Schleswig-Holstein, Lubeck, Germany.

Email: f.siemers@gmx.de

Received February 19 ${ }^{\text {th }}, 2012$; revised March 21 ${ }^{\text {st }}$ 2012; accepted April $10^{\text {th }}, 2012$

\begin{abstract}
Objective: Geriatric patients represent a separate group in the treatment of burn injury regarding pathogenesis, pathophysiology and therapeutic procedure. Methods: From 2000 to 2010 on our burn unit 53 patients aged over 60 years were treated. The mean age of the 27 women and 26 men was 76 years (60 - 102 years), with an average of $33.2 \%$ of total burn surface area. 25 patients (47.2\%) had severe inhalation injury, $77.4 \%$ of the patients suffered full thickness burn injuries. Mean ABSI Score was 12. Relevant additional diseases were coronary heart disease, heart insufficiency, hypertension, COPD, adiposity and chronic alcoholism. Results: 22 patients were treated by early operative intervention (day 0 - 4), 13 of these patients survived further process. In 17 cases first operative procedure could take place only at a later date, 14 patients deceased before operation. 26 patients developed pneumonia. The total morbidity amounted to 26 of 53 patients (49\%). A total of 19 injured with a burn index over 80 survived. 9 of them had an index of more than 100. Usual indices as the "Zellweger Index" and the ABSI-Score are not adequate to determine the prognosis of thermal in- juries in the elderly. Conclusion: The treatment of elderly with burn trauma is affected by many factors. Compared to younger patients the increased incidence of an inhalation injury is decisive for the prognosis of older patients. The frequency of pre-existing diseases is clearly largely than with younger burn injured patients. These prognostically relevant parameters were not sufficiently considered by established score systems.
\end{abstract}

Keywords: Burn Injury; Intensive Care; Elderly

\section{Introduction}

Literature referring to management of burn injuries in elderly is very rare. From more than 30,000 publications about therapy and results of the treatment of extensive burn injuries over the last ten years only 18 publications deal with this special topic. In a 10 year retrospective analysis of therapy and results of treatment of elderly patients following extensive burn injuries in our clinic were analysed.

The study should evaluate, if the "Zellweger Index" [1], which is known as prognostic score in Europe, is applicable to this group of patients. In this paper we are going to present the characteristics of the patients with an age of more than 60 years were treated in the burn unit by (age, sex, thickness of the burn injury, inhalation trauma, ABSI score, addidtional diseases); the therapy prossess of the burn patients (wound dressing, surgery, blood parameters microbiology results). Beyond in history we want to clarify whether additional diseases and the special therapy management represent a substantially more important role for successful treatment with extensively burned injured older patients, as so far accepted.

\section{Patients and Method}

\subsection{Patients Characteristics}

Between 2000 and 2010, 53 patients with an age of more than 60 years were treated in the burn unit at the University Hospital Schleswig-Holstein, Campus Luebeck. The average age of the 27 women and 26 men amounted to 76 years (60 - 102 years), in average $33.2 \%$ of the body surface (5\% - 95\%) was affected by thermal injury. 18 female and 17 male patients suffered their injuries by flames, 9 women and 9 men suffered scald injuries. 41 patients had full thickness thermal injuries (77.4\%), 11 patients (20.8\%) sustain deep partial thickness burn injuries. 25 patients (47.2\%) suffered severe inhalation injury, diagnosed by bronchoscopy. The average body core temperature at time of admission was $35.7^{\circ} \mathrm{C}$ degree $\left(31.0^{\circ} \mathrm{C}-37.8^{\circ} \mathrm{C}\right)$. The mean time between accident and admission in our burn unit was 25.5 hours.

The patients were assessed by their admission using the Zellweger Index and ABSI Score (Abbreviated burn severity index). The Zellweger Index represents a prognosis in lethality and is calculated by adding the burn 
injury TBSA in \% with the age of the patients. The lower the burn index is beneath 80 the better the chance of survival is. The ABSI is a simple and clinically useful score that is derived from multivariete logistic regression. This score, consisting of five variables (sex, age, presence of inhalation trauma, presence of full thickness burn, TBSA) demonstrates predective power for classifying patients according to their risk (Table 1).

On admission the average ABSI Score [2] (Figure 1) was 12. The average burn index (total burn surface area + age) [1] (Figure 1) was 108.6. 19 patients with a burn index over 80 survived. From these 19 survivors 9 patients had an index of more than 100. Relevant additional diseases were coronary heart disease $(n=32)$, heart insufficiency NYHA III - VI $(\mathrm{n}=27)$, hypertension $(\mathrm{n}=$ 25), COPD $(n=11)$, adiposity with a BMI $>30(n=14)$ and chronic alcoholism $(\mathrm{n}=9)$.

\subsection{Therapy Process}

At admission in our burn unit the first treatment took place in the resuscitation room. After stabilisation of

Table 1. (a) The ABSI-Score tries to make a prognosis for the probability of death by taking into account Age, Gender, TBSA and other Injuries or Illness (Tobiasen 1982) [2]; (b) The ABSI-score and the prognosis.

(a)

\begin{tabular}{|c|c|c|}
\hline \multicolumn{3}{|c|}{ ABSI-SCORE (abbreviated burn severity index) [2] } \\
\hline Parameters & & score \\
\hline \multirow{10}{*}{ TBSA (\%) } & $1-10$ & 1 \\
\hline & $11-20$ & 2 \\
\hline & $21-30$ & 3 \\
\hline & $31-40$ & 4 \\
\hline & $41-50$ & 5 \\
\hline & $51-60$ & 6 \\
\hline & $61-70$ & 7 \\
\hline & $71-80$ & 8 \\
\hline & $81-90$ & 9 \\
\hline & $91-100$ & 10 \\
\hline \multirow[t]{5}{*}{ Age (years) } & $0-20$ & 1 \\
\hline & $21-40$ & 2 \\
\hline & $41-60$ & 3 \\
\hline & $61-80$ & 4 \\
\hline & $>80$ & 5 \\
\hline \multirow[t]{5}{*}{ Other parameters } & Male & 0 \\
\hline & Female & 1 \\
\hline & Inhalation trauma & 1 \\
\hline & 3 burn & 1 \\
\hline & Severe Illness & 1 per illness \\
\hline \multicolumn{3}{|c|}{ (b) } \\
\hline Total score & \multicolumn{2}{|c|}{ Probability of death (\%) } \\
\hline $2-3$ & \multicolumn{2}{|c|}{$<1$} \\
\hline $4-5$ & \multicolumn{2}{|c|}{$>2$} \\
\hline $6-7$ & \multicolumn{2}{|c|}{$10-20$} \\
\hline $8-9$ & \multicolumn{2}{|c|}{$30-50$} \\
\hline $10-11$ & \multicolumn{2}{|c|}{$60-80$} \\
\hline$>11$ & \multicolumn{2}{|c|}{$>80$} \\
\hline
\end{tabular}

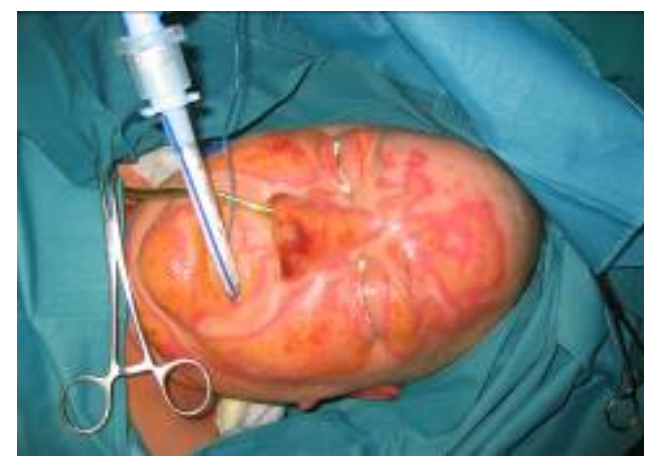

(a)

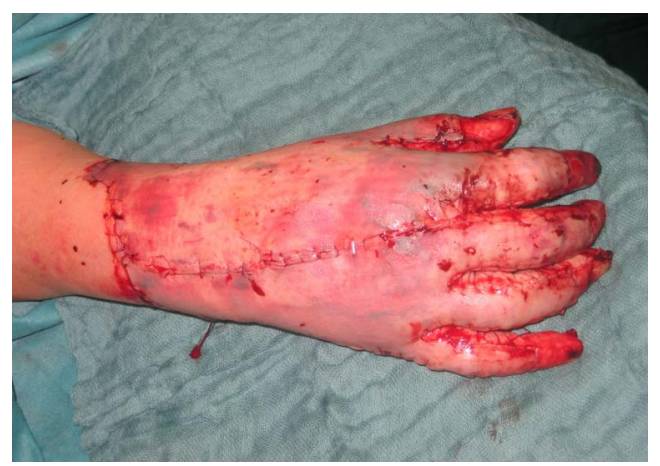

(b)

Figure 1. (a) and (b) 64-years old male, suffered deep partial thickness burn injuries of the head and the hands during by a fire accident at home.

airways, ventilation and circulation, blisters, dead epithetlium and the remnants of burned clothes were removed. The wounds were rinsed with an antiseptic solution (polyhexanid, Lavasept ${ }^{\circledR}$ ). In cases with circumferential full thickness thermal injuries relaxation incisions (escharotomy) were performed. With conclusion of the initial treatment the injuries were covered by a wound dressing consisting of polyhexanid cream (Lavasept ${ }^{\circledR}$ ) and wound gaze $\left(\right.$ Adaptic $^{\circ}$ ). This was followed by intensive care treatment with a balanced fluid therapy. Due to postburn processes in the first 24 hours following trauma such as "capillary leak” a progressive hypovolaemia makes an adequate fluid therapy up to 40 litres necessary. After decrease of the replacement volume fluid a second period with mobilisation of the interstitial fluid follows. This mobilisation is supported by the use of colloids as well as forced diuresis. If necessary a continuous veno-venous Haemodiafiltration (CVVHDF) is used during this period. After further 24 to 48 hours edemas are usually so far regressed that, presupposed a stable pulmonal and hemodynamic situation, surgical therapy (early necrectomy) can be begin.

Deep partial thickness burn injuries were treated tangential excision, full thickness thermal injuries by epifascial necrectomy. After debriding the defect areas were covered by skin grafts. Partial thickness and superficial 
thermal injuries healed without surgical procedures, using occlusive dressings (i.e. hydrocolloid dressings).

First surgical procedures took place 5.48 days $(1-27$ days) in average following trauma. In 22 cases early necrectomy (0 - 4 days after accident) was accomplished. With 17 patients the first surgical procedure took place later, 13 patients were not operated. In the further clinical process altogether 26 patients (49\%) developed pneumonia. In the group of patients who underwent early necrectomy 12 (54.5\%) developed pneumonia, in the cases with late necrectomy 11 patients (61.1\%) developed pneumonia (Figures 2 and $\mathbf{3}$ ).

Regarding the laboratory parameters leukocytes showed an early rise followed by a decrease at day 7 . In the following 6 days leukocytes rise to higher value (Figure 4). Fibrinogen rise from a normal value on the first day of treatment to a maximum at day 7. Later the value of fibrinogen decreased slowly. C-reactive protein (CRP) increased from day 1 to 7 (maximum $154.75 \mathrm{mg} / \mathrm{dl}$ ). Up to day 13 a decrease to an average value of $104.43 \mathrm{mg} / \mathrm{dl}$ was observed.

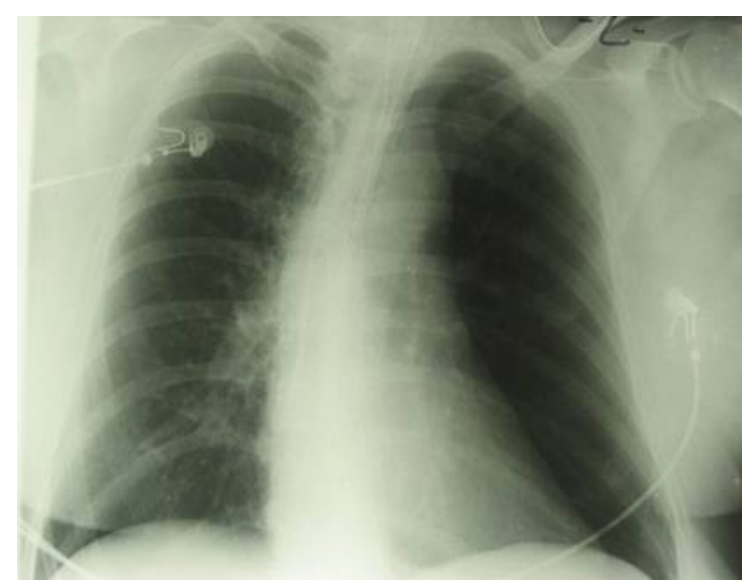

(a)

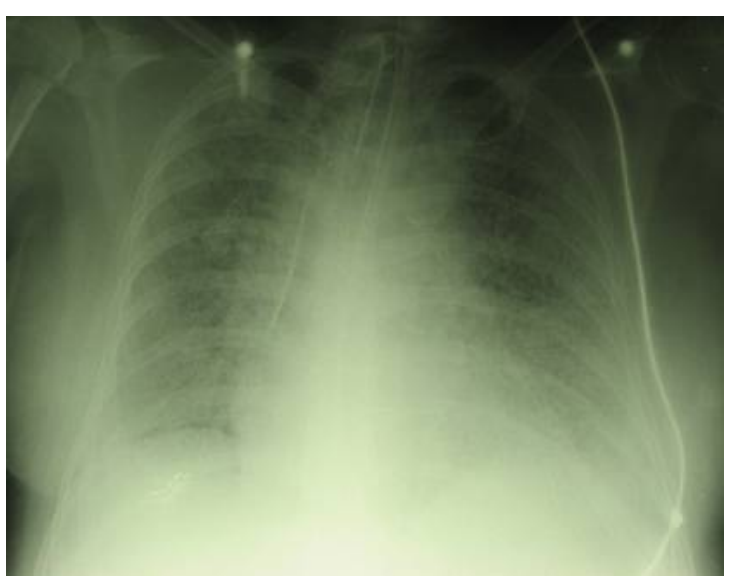

(b)

Figure 2. (a) Chest $x$-ray at admisson: no pathological findings, no signs for inhalative trauma; (b) Chest $x$-ray at day 11: interstitial infiltrates compromises the whole lung.

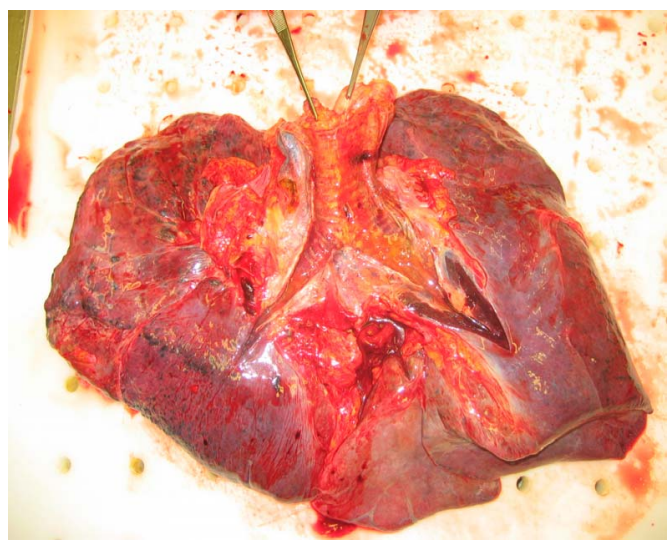

Figure 3. Obduktion following death at day 12: fibrosis of the whole parenchyma, signs for a beginning pneumonia.

During the further course different bacteria could be isolated (multiple nomination possible) from the burn wounds. In 11 cases (20.7\%) retrospectively no data could be acquired concerning this question. In 22 cases (41.5\%) escherichia coli and with 33 patients (62.3\%) staphylococcus aureus were identified on the wounds. Further isolated organisms were coagulase negative staphylococcus aureus $(\mathrm{n}=19$; 35.8\%), pseudomonas aeruginosa $(\mathrm{n}=13$; $24.5 \%)$, acinetobacter baumanii $(n=6 ; 11.3 \%)$, enterobacter species ( $\mathrm{n}=21 ; 39.6 \%)$, candida albicans $(\mathrm{n}=18$; $34,0 \%)$, candida glabrata $(n=6 ; 11.3 \%)$, as well as aspergillus in one case (1.9\%). Following the specification of the microorganisms, a targeted systemic antibiotic therapy by one or more sensitive products was performed. Antibiotic therapy was continued for 4.83 days in average.

Early systemic hemodynamic changes made a medical support by inotropes necessary in several patients. 37 patients received dopamine, in 19 cases in addition adrenaline was necessary, 11 patient received noradrenaline. 10 patients circulation was supported by dobutamine.

The acute oliguric renal failure, respectively a threatening renal failure due to increased myoglobin which occurs in cases with rhabdomyolisis, represents a serious complication in the treatment of major burn injuries. 7 patients (13.2\%) were treated precautionary or curatively by continuous veno-venous haemodiafiltration (CVVHDF).

\subsection{Statistical Analysis}

Variables were analyzed using Man-Whitney-U test. Pvalues lower than 0.001 were regarded as statistical significant.

\subsection{Results}

A total of 19 injured with a burn index over 80 survived. of 19 survivors 9 patients had an index of more than 100 . The burn index differed significantly $(\mathrm{p}<0.001)$. The value of the ABSI Score differed between survivors an deceased patients with $\mathrm{p}<0.001$. 


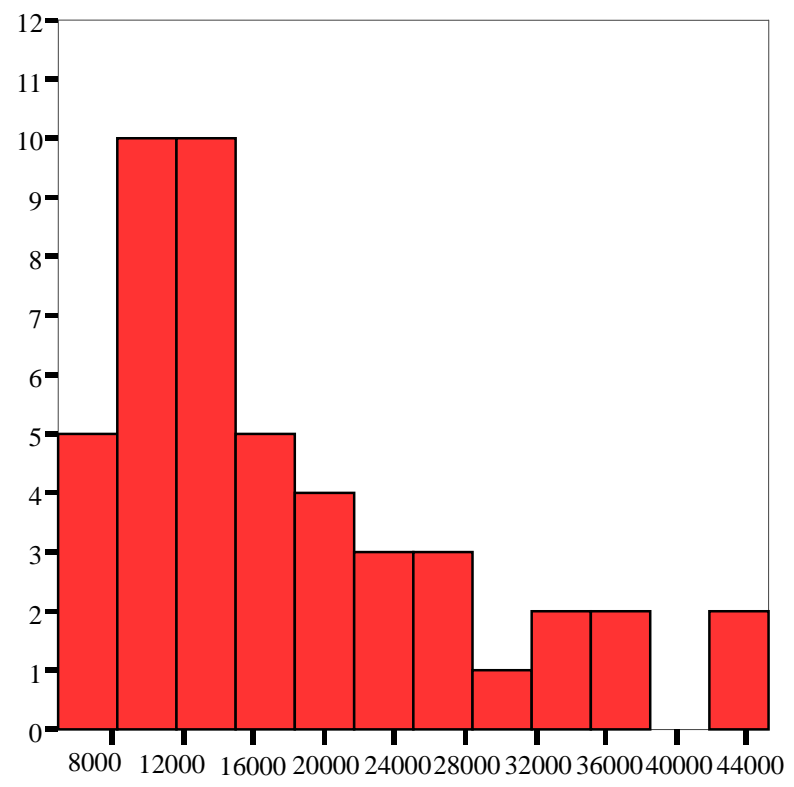

(a)

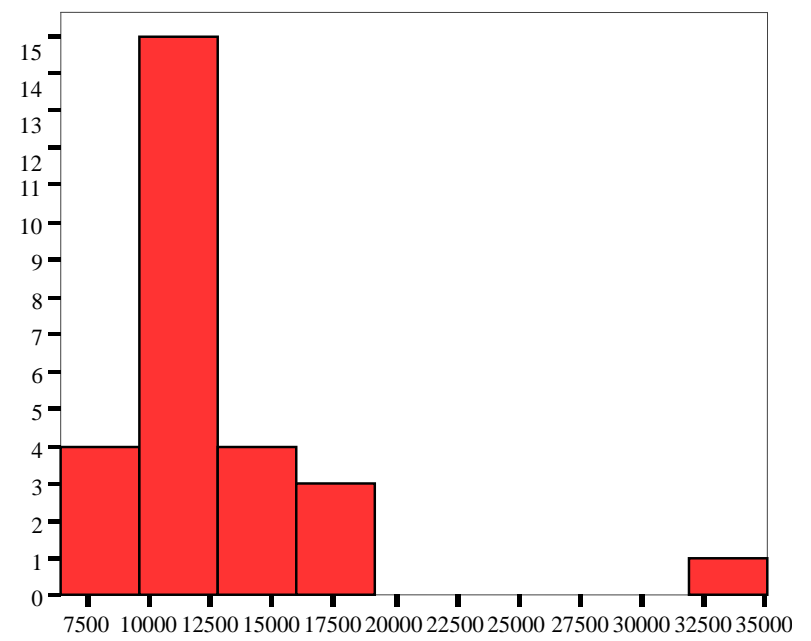

(c)

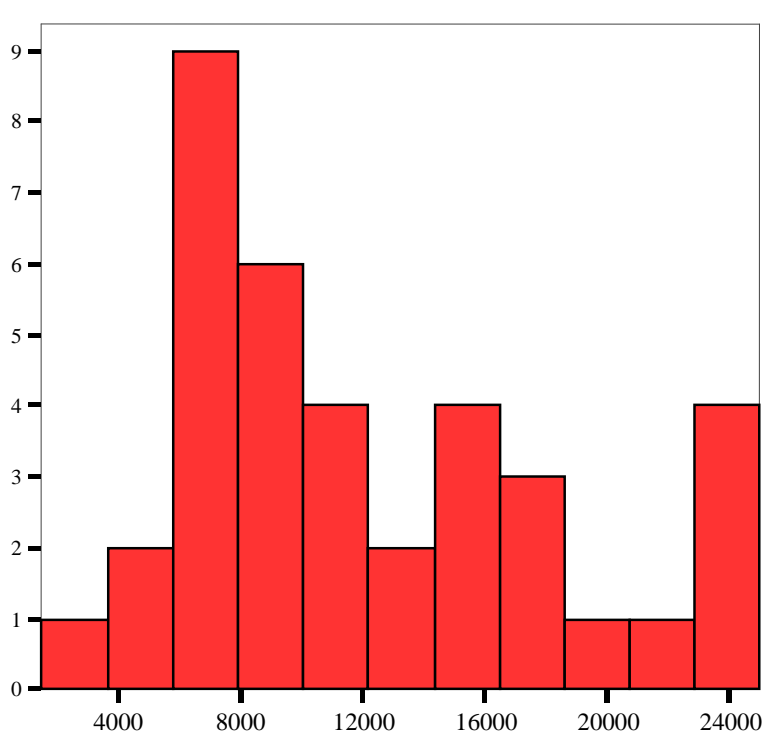

(b)

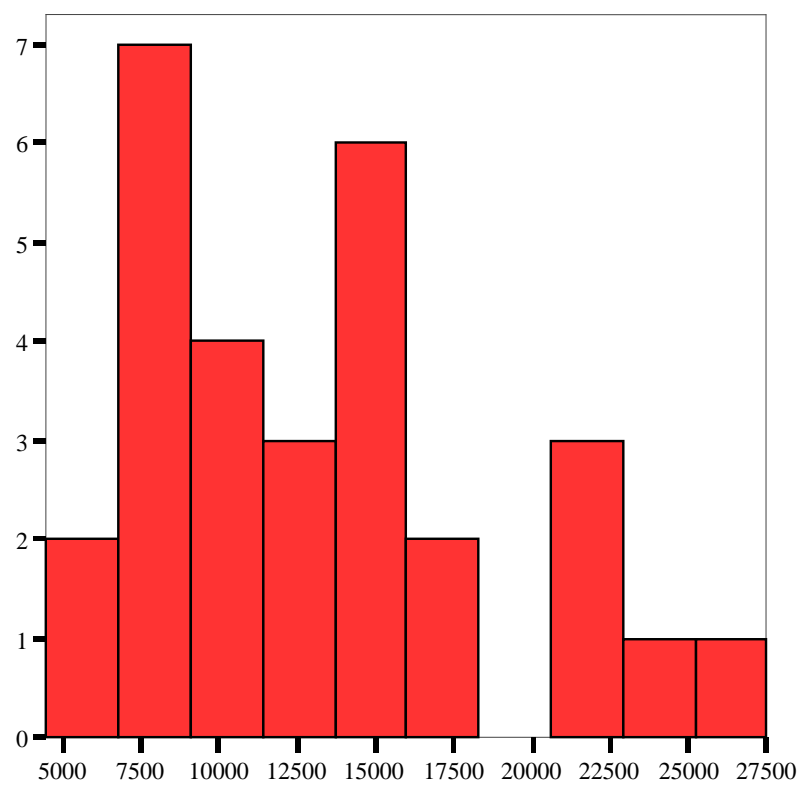

(d)

Figure 4. (a)-(d) These tables represent the correlation between the number of patients and leucocytes on 1st, 7th, 10th and 13th day after the injury. Leukocytes showed an early rise followed by a decrease at day 7. In the following 6 days leukocytes rise to higher value.

\section{Discussion}

In all age groups males were more susceptible to thermal injuries than females, in average by a factor of about two. In the group of older patients ( $>60$ years old) this relationship shifted to a ratio of $1: 1$. These changes could be explained by hazardous behaviour for example by handling with flammable liquids. Additionally in younger persons another common cause for burn injuries resulted from work-related accidents: With increasing age the rate of the domestic accidents rise in the group of both sexes, not least by the increasing frailty and clumsiness too [3-5]. The portion of burn injuries was twice as high as the number of scald injuries. Burn injuries resulted often because of carelessness, e.g. falling asleep with a burning cigarette, alcohol abuse or by other domestic accidents $[5,6]$.

18 patients suffered scald injuries, frequently caused 
by a syncope while taking a bath or a shower [5]. In old people home's water temperature in the hot water heater is often raised up to 70 degrees Celsius as a protection measure against pneumonia legionella [7]. Accidental contact to this hot water often induces full thickness thermal injuries. This kind of injury is disproportionally associated with elderly patients (77.4\%). This circumstance can be explained by a longer exposition time due to of immobility. Additionally the specific changes of dermal structures in elderly are crucial. The thickness of the skin decreases through increasing skin atrophy in older patients and anatomical structures like the subdermal vascular structures which are affected by typical agerelated diseases like diabetes or arterial occlusive disease. These circumstances delay important processes for regeneration of the skin. This may lead to deeper thermal damaging as diagnosed on admission [8]. Nearly half $(47.2 \%)$ of the patients suffered an inhalation injury. This incidence lies clearly above younger patients (19.6\%) $[4,9]$.

Elderly patients often cannot rescue themselves from the site of accident because of increasing immobility. The longer exposition in flames gases, resulting from it, explains the increased incidence of inhalation injury. In many cases the process was made more difficult by e.g. an affected pulmonal system based on a COPD (20.8\%). An inhalation injury will have been under these circumstances a determining factor for the bad prognosis in many of the patients. 17 (65.4\%) of the 26 deceased patients (65.4\%) had an inhalation injury [4].

Especially in the first hours of emergency treatment of thermally injured patients the management of body temperature is of particular importance. Body temperature measured intravesically at admission was in average 35.7 degrees Celsius (31.0 - 37.8 degrees Celsius). Feared hypothermia leads to well-known further complications such as cardiac dysrhythmia to a centralization of circulation. This causes lower perfusion of the periphery with delay regeneration processes. Furthermore hypothermia leads to decreased blood levels of adenosintriphosphate with high risk for complications such as organ failure [10]. An important factor influencing the further course of burn-injured patients is comorbidity. 27 patients with cardiac insufficiency (NYHA III - IV, 50.9\%) were exposed to a high volume loading with increased risk for a pulmonary edema, due to the initially very large volume needed. In such situations, there is always high risk of pulmonary edema even without coexistence of inhalation injury an pulmonal decompensation under artificial ventilation occur. In these situations a great danger arises from applicated catecholamines. Due to the intravascular leak of volume an decrease cardiac load with tachycardia and underperfusion of the heart muscle can occur, especially in patients with coronary heart disease $(n=32$,
60.4\%). In the treatment of burn injured patients with low cardiac output an adapted volume substitution is necessary. This can be done in relation to haematocrit level and hemodynamic situation in combination with administration of dobutamine. Because of its positive inotropic effect on the heart muscle and low vasocontrictive effect in the peripheral vascular system it is especially suitable for usage with burn injured patients. In general catecholamines should be applied restrictively in burn injured patients because of vasoconstrictive effect in the soft tissue.

If it is not possible to establish adequate blood pressure by fluid substitution because of capillary leak, catecholamines must be substituted. Following stabilization of the hemodynamic situation, problems because of vasoconstriction in the capillary system arise. Through this, the initial depth of the thermal injury can increase. In these cases surgical treatment with skin grafting could become necessary. This process is known as afterburning. Even in the further clinical process, the use of catecholamines needs critical evaluation and discussion as the use of these drugs can e.g. endanger skin graft integration.

In comparison to our patients with an age under 60 years we saw an increase of co morbidity. However severe courses of burn trauma, depth of the thermal injury, size of injured body surface and inhalation injury increase in elderly significantly. The frequent cardiopulmonal instability does not permit an early but desirable surgical treatment. Burn injuries in elderly represent a serious trauma that makes an early transfer to a burn unit necessary.

Hoerbrand [11] called attention to the importance of serious diseases in burn patients independent of the age of the patients. Additional diseases as heart insufficiency, coronary heart disease, COPD, diabetes mellitus, hypertension and chronic alcoholism represent a crucial prognostic factor.

In a current publication Alden [12] refers to the coincidence of dementia and burn injury in elderly. However the course following the trauma showed no significant influences through dementia. In our study we had the same findings.

An early necrectomy within the first 4 days following burn injury was performed in 22 patients (41.5\%). In all of these cases an autological skin grafting following tangential excision or an epifascial necrectomy was achieved. 13 of these patients (59.1\%) with an average burn index of 90.8 survived in further process. The advantage of the early, definite surgical procedure is the shorter duration of the immobilization and hospitalisation. This also had an influence on the development of pneumonia. 12 patients (54.5\%) of the group with an early surgical therapy and 11 burn injured (61.1\%) with later operations suffered pneumonia. 
Due to regular microbiological examinations with resistance screening an early adapted antibiotic therapy could be introduced. This therapy started at an average of 4, 8 days in combination with an intensive topical application of antiseptics at the wound surface. In comparison to other burn units the proof of hospital acquired bacteria like pseudomonas aeruginosa was noticeably less.

In 2002 Oncul [13] described a contamination rate by pseudomonas aeruginosa of $41.7 \%$ of the treated patients on an burn unit. Estahbanati [14] even reported a contamination rate of $57 \%$. In our study we had an evidence of $24.5 \%$ (13 patients). Most frequently we found staphylococcus aureus (33 patients, 62.3\%) and escherichia coli (22 patients, 41.5\%). In no case methicillin resistant Staphylococcus aureus (MRSA) was found. Oncul [13] reported with MRSA infections within 25\% of his patients.

On the day of admission in 40 patients (75.5\%) we found a leukocytosis with a mean level of $17590 / \mathrm{ml}$ (range 4920 - 45270/ml). A possible explanation could be a trauma associated release of endogenous stress hormons like cortisol. The consequence is a release of granulocytes from the vascular endothelium [15]. During further course the endogenous hormone releases fades away. This could be the explanation for an decrease of the leukocytes value on day 4 and 7 (on both days on an average approx. 11000/ml). Within the immune response there was an increase of the leukocytes to level of $13093 / \mathrm{ml}$ at day 10 . However there is great influence on this value by e.g. wound infection, pneumonia or urinary tract infection. On day 13 the value of leukocytes decreased to an average of $12434 / \mathrm{ml}$, explained by successful healing process of the wound ground following surgical therapy. This observation correlates with the development of the fibrinogen level which had risen on day 7 due to the immune response to an average value of $7.22 \mathrm{~g} / \mathrm{l}$ (range 2.05 - $17.40 \mathrm{~g} / \mathrm{l}$ ). Until day 13 the fibrinogen level had fallen to an mean value of $6.36 \mathrm{~g} / \mathrm{l}$ (range 2.84 - $8.98 \mathrm{~g} / \mathrm{l}$ ).

Also the C-reactive protein (CRP), which among others is responsible for complement activation and the fixation of freed bacterial DNA, showed a similar process [16]. In the context of the immune response the rise of the CRP level was highest between day 4 and 7 following the trauma. The average maximum at day 7 was 154.75 $\mathrm{mg} / \mathrm{dl}$ (7 - $307 \mathrm{mg} / \mathrm{dl})$. Up to day 13 the value declined to $104.43 \mathrm{mg} / \mathrm{dl}$ (16 - $274 \mathrm{mg} / \mathrm{dl})$.

The use of the Zellweger-Index [1] suggests that the sum of age of the patient and total burn surface area (TBSA) in per cent represents a lethal prognosis at a value of over 100 . The great disadvantage of this score system is that relevant parameters as pre-existing diseases, inhalation trauma burn depth are not considered.

If a patient with severe pre-existing diseases suffers a burn injury, this can affect the prognosis of the patient crucially despite only a small burned body surface area. However inhalation injury can often be crucial for the prognosis. The Zellweger-Index [1] should not be considered valid for all patients. This recommendation reflects the results of our examinations, with an average burn index of 108.6. Generally a value of more than 100 points applies take as prognostically infaust. This is also the case with the ABSI-Score [2] which does not account for relevant diseases and inhalation injury. In the use of the ABSI Score the severe complication of inhalation injury is only computed with one score point, comparable with one pre-existing disease, female sex or the existence of full thickness burn injury. As already described, we had to state that an inhalation injury represents a crucial factor in the prognosis of burn injured patients also during low-grade burn injury.

\section{Conclusion}

The treatment of elderly with burn trauma is affected by many factors, which have little relation to burn or scald injury. Compared to younger patients the increased incidence of an inhalation injury is decisive for the prognosis of older patients. On the other hand the frequency of pre-existing diseases e.g. coronary heart disease, heart insufficiency, COPD, diabetes mellitus, in this group of patients is clearly largely than with younger burn injured patients. These prognostically relevant parameters were not sufficiently considered by established score systems like Zellweger-Index or ABSI Score. The poor prognosis for burn injured patients with a Zellweger Score more than 100 is disproved by the results in our patient group. Patients (17\%) with an index of over 100 survived their burn or scald trauma.

\section{REFERENCES}

[1] G. Zellweger, "Die Behandlung der Verbrennungen, Praktische Hinweise für Diagnose, Therapie, Rehabilitation," German Medical Company, 2nd Edition, Auflage, Colognia, 1985.

[2] J. Tobiasen, J. M. Hiebert and R. F. Edlich, "The Abbreviated Burn Severity Index,” Annals of Emergency Medicine, Vol. 11, No. 5, 1982, pp. 260-262. doi:10.1016/S0196-0644(82)80096-6

[3] W. S. Ho, S. Y. Ying and H. A. Chan, "A Study of Burn Injuries in the Elderly in A Regional Burn Centre,” Burns, Vol. 27, No. 4, 2001, pp. 382-385. doi:10.1016/S0305-4179(00)00146-7

[4] P. Q. Bessey, R. R. Arons, C. J. Dimaggio and R. W. Yurt, "The Vulnerabilities of Age: Burns in Children and Older Adults,” Surgery, Vol. 140, No. 4, 2006, pp. 705-715.

[5] A. R. Ehrlich, "Preventing Burns in Older Patients," American Family Physician, Vol. 74, No. 10, 2006, pp. 1688-1693.

[6] V. McGill, A. Kowal-Vern and R. L. Gamelli, "Outcome 
for Older Burn Patients,” Archives of Surgery, Vol. 135, No. 3, 2000, pp. 320-325. doi:10.1001/archsurg.135.3.320

[7] W. L. Straus, J. F. Plouffe, T. M. File, H. B. Lipman, B. H. Hackman, S. J. Salstrom, R. F. Benson and R. F. Breiman, "Risk Factors for Domestic Acquisition of Legionnaires Disease. Ohio legionnaires Disease Group," Archives of Internal Medicine, Vol. 156, No. 15, 1996, pp. 1685-1692. doi:10.1001/archinte.1996.00440140115011

[8] B. McCampbell, N. Wasif, A. Rabbitts, L. Staiano-Coico, R. W. Yurt and S. Schwartz, "Diabetes and Burns: Retrospective Cohort Study," Journal of Burn Care \& Rehabilitation, Vol. 23, No. 3, 2002, pp. 157-166. doi:10.1097/00004630-200205000-00004

[9] D. L. Smith, B. A. Cairns, F. Ramadan, J. S. Dalston, S. M. Fakhry, R. Rutledge, A. A. Meyer and H. D. Peterson, "Effect of Inhalation Injury, Burn Size, and Age on Mortality: A Study of 1447 Consecutive Burn Patients," Journal of Trauma and Acute Care Surgery, Vol. 37, No. 4, 1994, pp. 655-659. doi:10.1097/00005373-199410000-00021

[10] A. Seekamp, M. van Griensven, F. Hildebrandt, T. Wahlers and H. Tscherne, "Adenosine-Triphosphate in Trauma-Related and Elective Hypothermia," Journal of Trauma, Vol. 47, No. 4, 1999, pp. 673-683.

[11] F. Hörbrand, C. Schrank, G. Henckel-Donnersmarck and W.
Muhlbauer, "Integration of Preexisting Diseases and Risk Factors in the Abbreviated Burn Severity Index (ABSI)," Anasthesiol Intensivmed Notfallmed Schmerzther, Vol. 38, No. 3, 2003, pp. 151-157.

[12] N. E. Alden, A. Rabbitts and R. W. Yurt, "Burn Injury in Patients with Dementia: An Impetus for Prevention,” Journal of Burn Care \& Rehabilitation, Vol. 26, No. 3, 2005, pp. 267-271.

[13] O. Oncul, F. Yuksel, H. Altunay, C. Acikel, B. Celikoz and S. Cavuslu, "The Evaluation of Nosocomial Infection During 1-Year-Period in the Burn Unit of a Training Hospital in Istanbul, Turkey,” Burns, Vol. 28, No. 8, 2002, pp. 738744. doi:10.1016/S0305-4179(02)00106-7

[14] H. K. Estahbanati, P. P. Kashani and F. Ghanaatpisheh, "Frequency of Pseudomonas Aeruginosa Serotypes in Burn Wound Infections and Their Resistance to Antibiotics," Burns, Vol. 28, No. 4, 2002, pp. 340-348. doi:10.1016/S0305-4179(02)00024-4

[15] J. A. Griswold, "White Blood Cell Response to Burn Injury," Seminars in Nephrology, Vol. 13, No. 4, 1993, pp. 409-415.

[16] G. Uhlenbruck, J. Solter, E. Janssen and H. Haupt, "Two new, Additional 'Combining Sites' of C-Reactive Protein: Lectin Specificity of the Anti-Galactan Type and AntiHaemocyanin Reactivity," Hoppe Seylers' Journal of Physiological Chemistry, Vol. 362, No. 8, 1981, pp. 1167-1169. 